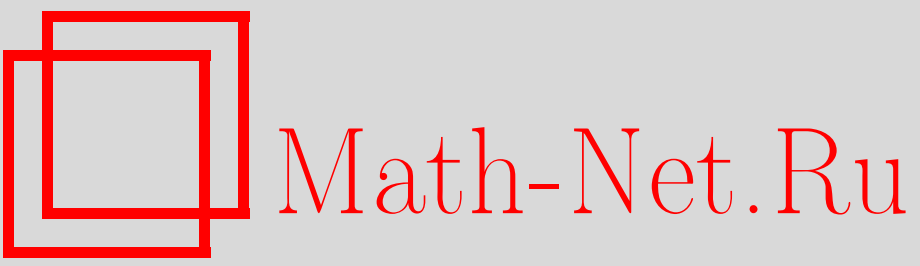

K. В. Полякова, Нормали высшего порядка на многообразии, Итоги науки и техн. Сер. Соврем. мат. и ее прил. Темат. обз., 2020, том 180, 85-90

DOI: https://doi.org/10.36535/0233-6723-2020-180-85-90

Использование Общероссийского математического портала Math-Net.Ru подразумевает, что вы прочитали и согласны с пользовательским соглашением

http://www.mathnet.ru/rus/agreement

Параметры загрузки:

IP : 3.82 .47 .9

26 апреля 2023 г., 14:32:31 


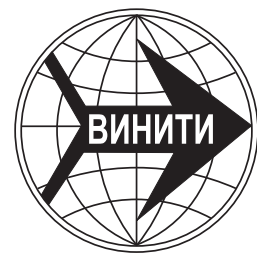

ИТОГИ НАУКИ И ТЕХНИКИ.

Современная математика и ее приложения.

Тематические обзоры.

Том 180 (2020). C. 85-90

DOI: $10.36535 / 0233-6723-2020-180-85-90$

УДК 514.76

\title{
НОРМАЛИ ВЫСШЕГО ПОРЯДКА НА МНОГООБРАЗИИ
}

\author{
(c) 2020 г. $\quad$ К. В. ПОЛЯКОВА
}

\begin{abstract}
АннотАция. На $n$-мерном гладком многообразии рассмотрены нормали высших порядков двух видов, т.е. пространства, дополняющие касательное пространство порядка 1 или $r-1$ до касательного пространства порядка $r$. Показано, что производные одних базисных векторов по направлению данных базисных векторов первого (второго) порядка равны значениям дифференциалов первого (второго) порядка первых векторов на данных векторах. С помощью дифференциалов базисных касательных векторов первого и второго порядков построены отображения из множества касательных векторов первого порядка во множество векторов нормалей второго и третьего порядков. Заданы отображения, порождающие горизонтальные векторы второго и третьего порядков для канонической аффинной связности первого и второго порядков соответственно.
\end{abstract}

Ключевые слова: дифференциальная форма, касательное пространство, нормаль на многообразии, аффинная связность.

\section{HIGHER-ORDER NORMALS ON MANIFOLDS}

\author{
(c) 2020 K. V. POLYAKOVA
}

\begin{abstract}
On an $n$-dimensional smooth manifold, we consider higher-order normals of two types, i.e., the spaces that complement the tangent space of orders 1 or $r-1$ to the tangent space of order $r$. We prove that the derivatives of some basic vectors in the direction of the given first-order (second-order) basis vectors are equal to the values on these vectors of the first-order (second-order) differentials of the first vectors. Using the differentials of basic tangent vectors of the first and second orders, we construct mappings from the set of first-order tangent vectors to the set of second- and third-orders normal vectors. Also, we introduce mappings that generate horizontal second- and third-order vectors for the canonical first- and second- order affine connections, respectively.
\end{abstract}

Keywords and phrases: differential form, tangent space, normal on a manifold, affine connection.

AMS Subject Classification: 53B05, 58A10

1. Введение. Данная работа относится к дифференциальной геометрии, а методика исследования основана на применении способа Г. Ф. Лаптева (см. $[7,8])$ задания связности в главных расслоениях и разработанного им метода продолжений и охватов, который обобщает метод подвижного репера и внешних форм Э. Картана. Применение метода Картана-Лаптева, оперирующего структурными уравнениями и деривационными формулами, позволяет эффективно использовать тангенциальнозначные формы, т.е. дифференциальные формы со значениями в касательных пространствах различных порядков. Указанные формы широко применяются во многих разделах современной физики (см., например, $[2,15]$.

Отправным пунктом для построения различных тангенциальнозначных форм на расслоении линейных реперов является каноническая форма на этом расслоении; связывая касательное и кокасательное пространства этого расслоенного многообразия, она соответствует тождественным 
отображениям этих пространств. При этом действия тангенциальнозначных форм имеют двойственный характер: они действуют как в пространстве касательных векторов к расслоению линейных реперов, так и в пространстве ковекторов. Все тангенциальнозначные формы, возникающие в результате продолжения (внешнего дифференцирования и последующего разрешения по лемме Э. Картана), дают возможность строить касательные пространства 2-го и более высоких порядков, кроме того, эти формы задают важные отображения как касательных, так и кокасательных пространств различных порядков. В частности, отображения, определяемые дифференциалами базисных касательных векторных полей, переводят касательные векторы в нормальные векторы (см. [13]), т.е. векторы второго порядка, дополняющие касательное пространство 1-го порядка до касательного пространства 2-го порядка.

Метод Лаптева, базируясь на операции внешнего дифференцирования обычных (а также тангенциальнозначных) форм, с применением координатного задания векторов и форм дает возможность шире использовать деривационные формулы на многообразии.

Каноническая форма 1-го порядка $\theta=\omega^{i} \varepsilon_{i}(i, j, k, \ldots=1, \ldots, m)$ на многообразии $X_{m}$ связывает касательное $T X_{m}=\operatorname{span}\left(\varepsilon_{i}\right)$ и кокасательное $T^{*} X_{m}=\operatorname{span}\left(\omega^{i}\right)$ пространства к этому многообразию в его текущей точке $M$, а также соответствует тождественным преобразованиям этих пространств, т.е. (см. [12])

$$
\theta=\mathrm{id}_{T X_{m}}, \quad \theta=\mathrm{id}_{T^{*} X_{m}} .
$$

Каноническая форма является тензорнозначной (см. $[2,5])$, точнее, тангенциальнозначной. Дифференциальные 1-формы $\left\{\omega^{i}\right\}$ образуют кобазис, сопряженный подвижному базису $\left\{\varepsilon_{i}\right\}$, т.е. $\omega^{i}\left(\varepsilon_{j}\right)=\delta_{j}^{i}$. Фактически $\theta=\omega^{i} \otimes \varepsilon_{i} \in T^{*} X_{m} \otimes T X_{m}$, но значок тензорного умножения в форме $\theta=\omega^{i} \varepsilon_{i}$ будем опускать.

Реализуем подход, связанный с координатным выражением базисных и слоевых форм, а также векторов репера в слоях. Рассмотрим некоторую окрестность $m$-мерного многообразия $X_{m}$, в которой текущая точка определяется локальными координатами $x^{i}$. Слоевые координаты $x_{j}^{i}$, $x_{j k}^{i}, x_{j k l}^{i}$ на многообразии $X_{m}$ (см. [8, с. 149]) удовлетворяют соотношениям $\operatorname{det}\left(x_{j}^{i}\right) \neq 0, x_{k}^{i} x_{j}^{k}=\delta_{j}^{i}$ и симметричны по нижним индексам. При этом базисные $\omega^{i}$ и слоевые $\omega_{j}^{i}, \omega_{j k}^{i}$ формы имеют вид

$$
\begin{aligned}
\omega^{i} & =x_{j}^{i} d x^{j}, \\
\omega_{j}^{i} & =-x_{j}^{*} d x_{k}^{i}-x_{j k}^{i} \omega^{k}, \\
\omega_{j k}^{i} & =d x_{j k}^{i}+x_{j k}^{l} \omega_{l}^{i}-x_{l k}^{i} \omega_{j}^{l}-x_{j l}^{i} \omega_{k}^{l}+\left(x_{j k}^{s} x_{s l}^{i}-x_{j k l}^{i}\right) \omega^{l} .
\end{aligned}
$$

Структурные уравнения совокупности базисных $\omega^{i}$ и слоевых $\omega_{j}^{i}, \omega_{j k}^{i}$ форм главного расслоения реперов 2-го порядка, построенного над многообразием $X_{m}$, находятся внешним дифференцированием форм (1) (см. [8]).

Относительно натурального (голономного) репера $\left\{\partial_{i}=\partial / \partial x^{i}, \partial_{i j}=\partial^{2} / \partial x^{i} \partial x^{j}\right\}$ векторы $\varepsilon_{i}$ и $\varepsilon_{i j}$ раскладываются по формулам (см. [9])

$$
\varepsilon_{i}=\stackrel{*}{x}_{i}^{j} \partial_{j}, \quad \varepsilon_{i j}=\stackrel{*}{x}_{i}^{k} \stackrel{*}{x}_{j}^{l} \partial_{k l}+x_{i j}^{k} \stackrel{*}{x}_{k}^{l} \partial_{l} .
$$

Пространство $T^{2} X_{m}=\operatorname{span}\left(\varepsilon_{i}, \varepsilon_{i j}\right)$ в текущей точке $M$ многообразия $X_{m}$ называется касательным пространством порядка 2 , а также соприкасающимся пространством порядка 1 (см., например, [13]). Слоевые формы интерпретируются как компоненты инфинитезимального перемещения векторного репера $\varepsilon_{i}, \varepsilon_{i j}$, удовлетворяющего деривационным уравнениям

$$
\begin{aligned}
d \varepsilon_{i} & =\varepsilon_{j} \omega_{i}^{j}+\omega^{j} \varepsilon_{i j}, \\
d \varepsilon_{i j} & =\omega_{i}^{k} \varepsilon_{k j}+\omega_{j}^{k} \varepsilon_{i k}+\omega_{i j}^{k} \varepsilon_{k}+\omega^{k} \varepsilon_{i j k},
\end{aligned}
$$

которые получены дифференцированием векторов (2).

Дифференцируя каноническую форму $\theta=\omega^{i} \varepsilon_{i}$ обычным образом, получим каноническую форму 2-го порядка на многообразии $X_{m}$ (см., например, $\left.[1,9,16]\right)$

$$
d \theta=\left(d \omega^{i}+\omega^{j} \omega_{j}^{i}\right) \varepsilon_{i}+\omega^{i} \omega^{j} \varepsilon_{i j}
$$


причем $d \theta \in\left(T^{2} X_{m}\right)^{*} \otimes T^{2} X_{m}$, поскольку

$$
d \omega^{i}+\omega^{j} \omega_{j}^{i}, \omega^{i} \omega^{j} \in\left(T^{2} X_{m}\right)^{*}, \quad \varepsilon_{i j} \in T^{2} X_{m} .
$$

Пространство $\left(T^{2} X_{m}\right)^{*}$, сопряженное касательному пространству 2-го порядка $T^{2} X_{m}$, называется кокасательным пространством 2-го порядка. Репер $\left\{\varepsilon_{i}, \varepsilon_{i j}\right\}$ и корепер $\left\{d \omega^{i}+\omega^{j} \omega_{j}^{i}, \omega^{i} \omega^{j}\right\} 2$-го порядка являются сопряженными. Условия сопряженности для произвольных (не являющихся натуральными) базиса и кобазиса 2-го порядка имеют вид

$$
\left(d \omega^{i}+\omega^{j} \omega_{j}^{i}\right)\left(\varepsilon_{k}\right)=\delta_{k}^{i}, \quad \omega^{i} \omega^{j}\left(\varepsilon_{k l}\right)=\frac{1}{2}\left(\delta_{k}^{i} \delta_{l}^{j}+\delta_{l}^{i} \delta_{k}^{j}\right), \quad\left(d \omega^{i}+\omega^{j} \omega_{j}^{i}\right)\left(\varepsilon_{k l}\right)=0, \omega^{i} \omega^{j}\left(\varepsilon_{k}\right)=0 .
$$

Известно (см. [17]), что $\left\{d^{2} x^{i}, d x^{i} d x^{j}\right\}$ - натуральный корепер (см. [6, с. 54], [4, с. 175]) кокасательного пространства 2-го порядка $\left(T^{2} X_{m}\right)^{*},\left\{\partial_{i}, \partial_{i j}\right\}$ - натуральный корепер касательного пространства 2-го порядка $T^{2} X_{m}$. Ненулевые условия сопряженности можно записать следующим образом:

При этом

$$
d^{2} x^{i}\left(\partial_{j}\right)=\delta_{j}^{i}, \quad d x^{i} d x^{j}\left(\partial_{k l}\right)=\frac{1}{2}\left(\delta_{k}^{i} \delta_{l}^{j}+\delta_{l}^{i} \delta_{k}^{j}\right)
$$

$$
\begin{gathered}
T X_{m} \subset T^{2} X_{m}, \quad\left\{\partial_{i}\right\} \in\left\{\partial_{i}, \partial_{i j}\right\} ; \\
T^{*} X_{m} \not \subset\left(T^{2} X_{m}\right)^{*}, \quad\left\{d x^{i}\right\} \notin\left\{d^{2} x^{i}, d x^{i} d x^{j}\right\} .
\end{gathered}
$$

Выражение обычного дифференциала канонической формы $\theta=\omega^{i} \varepsilon_{i}$ многообразия $X_{m}$ относительно натуральных репера и корепера 2-го порядка принимает следующий вид:

$$
d \theta=d^{2} x^{i} \partial_{i}+d x^{i} d x^{j} \partial_{i j}
$$

2. Нормали на многообразии. Нормалъю (см. $[13,14])$ порядка $r$ в точке $M$ многообразия называется такое подпространство $N^{r}$ соприкасающегося пространства $O^{r} X_{m}$, которое имеет пересечение нулевой размерности с пространством $O^{r-1} X_{m}$, и размерность которого является дополнительной по отношению к размерности $O^{r-1} X_{m}$, т.е.

$$
N^{r} \oplus O^{r-1} X_{m}=O^{r} X_{m} .
$$

Для определения порядка нормали будем использовать порядки касательных, а не соприкасающихся пространств, полагая, что соприкасающееся пространство $r$-го порядка - это касательное пространство $(r+1)$-го порядка, т.е. $T^{r+1} X_{m}=O^{r} X_{m}$. Кроме того, в качестве нормалей будем рассматривать также пространства, дополняющие пространство $T X_{m}$ (а не только пространство $\left.T^{r} X_{m}\right)$ до пространства $T^{r+1} X_{m}$.

Нормалъю порлдка $r$ касательного пространства $T X_{m}$ (многообразия $X_{m}$ ) в точке $M$ будем называть подпространство $N_{1}^{r}$, дополняющее пространство $T X_{m}$ до пространства $T^{r} X_{m}$, т.е.

$$
N_{1}^{r} \oplus T X_{m}=T^{r} X_{m}
$$

В частности, для пространства $T X_{m}$ имеем следующие две нормали $N_{1}^{2}$ и $N_{1}^{3}$ :

$$
N_{1}^{2} \oplus T X_{m}=T^{2} X_{m} ; \quad N_{1}^{3} \oplus T X_{m}=T^{3} X_{m}
$$

Нормаль $N_{1}^{r}$ можно представить в следующем виде: $N_{1}^{r}=T^{r} X_{m} \backslash T X_{m}$; тогда $N_{1}^{2}=T^{2} X_{m} \backslash T X_{m}$, $N_{1}^{3}=T^{3} X_{m} \backslash T X_{m}$.

Нормалью порядка $r+1$ касательного пространства $T^{r} X_{m}$ в точке $M$ будем называть подпространство $N_{r}^{r+1}$, дополняющее пространство $T^{r} X_{m}$ до пространства $T^{r+1} X_{m}$, т.е. (ср. [13])

$$
N_{r}^{r+1} \oplus T^{r} X_{m}=T^{r+1} X_{m}
$$

Например, для пространства $T^{2} X_{m}$ имеем нормаль $N_{2}^{3}=T^{3} X_{m} \backslash T^{2} X_{m}$.

Если в равенстве $\varepsilon_{j}(f)=d f\left(\varepsilon_{j}\right)$ заменить функцию $f$ векторами $\varepsilon_{i}$, то будет иметь место следующая лемма.

Лемма 1. Для базисных касательных векторов 1-го порядка $\varepsilon_{i}$ справедливо равенство $d \varepsilon_{i}\left(\varepsilon_{j}\right)=\varepsilon_{j}\left(\varepsilon_{i}\right)$. 
При линейном отображении $d \varepsilon_{i}$, определяемом соотношением (3), имеем

$$
d \varepsilon_{i}\left(\varepsilon_{j}\right)=\varepsilon_{k} \omega_{i}^{k}\left(\varepsilon_{j}\right)+\varepsilon_{i k} \omega^{k}\left(\varepsilon_{j}\right)=\dot{\varepsilon}_{i j} .
$$

Кроме того,

$$
\varepsilon_{j}\left(\varepsilon_{i}\right)=\partial_{\varepsilon_{j}} \varepsilon_{i}=\partial_{x_{j}^{*} \partial_{l}}\left(\stackrel{*}{x} \partial_{i} \partial_{k}\right)=\stackrel{*}{x}_{i}^{k} \stackrel{*}{x}_{j}^{l} \partial_{k l}=\varepsilon_{i j}-x_{i j}^{k} \varepsilon_{k}=\dot{\varepsilon}_{i j} .
$$

Векторы $\dot{\varepsilon}_{i j}$ удовлетворяют уравнениям $\Delta \dot{\varepsilon}_{i j}=\dot{\varepsilon}_{i j k} \omega^{k}$, т.е. инвариантны в совокупности, причем $\dot{\varepsilon}_{i j k}=\varepsilon_{i j k}-x_{i j}^{l} \dot{\varepsilon}_{l k}-x_{i j k}^{l} \varepsilon_{l}$, где векторы

$$
\varepsilon_{i j k}=\stackrel{*}{x}_{i}^{l} x_{j}^{*} x_{k}^{*} \partial_{l s t}+x_{i j k}^{l} \varepsilon_{l}+\left({ }^{*}{ }_{l}^{p}{ }^{*}{ }_{j}^{q} x_{i k}^{l}+\stackrel{*}{x}_{i}^{p} x_{l}^{q} x_{j k}^{l}+\stackrel{*}{x}_{l}^{p} x_{k}^{*} x_{i j}^{l}\right) \partial_{p q}
$$

принадлежат касательному пространству 3-го порядка $T^{3} X_{m}$. Дифференциальный тензорный оператор $\triangle$ имеет вид (см., например, $[1,3,16])$

$$
\Delta t_{j k}^{i}=d t_{j k}^{i}+t_{j k}^{l} \omega_{l}^{i}-t_{l k}^{i} \omega_{j}^{l}-t_{j l}^{i} \omega_{k}^{l} .
$$

Линейные отображения

$$
d \varepsilon_{i}: T X_{m} \rightarrow N_{1}^{2}=T^{2} X_{m} \backslash T X_{m}
$$

определяют нормаль 2 -го рода $N_{1}^{2}=\operatorname{span}\left(\dot{\varepsilon}_{i j}\right)$ для пространства $T X_{m}$.

Базисные векторы $\dot{\varepsilon}_{i j}=\varepsilon_{i j}-x_{i j}^{k} \varepsilon_{k}$ нормали 2-го порядка $N_{1}^{2}$ являются:

(i) производными векторов $\varepsilon_{i}$ по их направлениям, т.е. $\dot{\varepsilon}_{i j}=\partial_{\varepsilon_{j}} \varepsilon_{i}$,

(ii) образами векторов $\varepsilon_{j}$ при отображениях $d \varepsilon_{i}$, т.е. $\dot{\varepsilon}_{i j}=d \varepsilon_{i}\left(\varepsilon_{j}\right)$.

Для касательных векторов $u=u^{i} \varepsilon_{i}, v=v^{i} \varepsilon_{i}$ справедливо:

$$
d u(v)=\left(\frac{\partial u^{j}}{\partial x^{k}} x_{i}^{k} \varepsilon_{j}+u^{j} \dot{\varepsilon}_{j i}\right) v^{i} .
$$

Теорема 1. Пусть и: $X_{m} \rightarrow T X_{m}$ - векторное поле, тогда du: $T X_{m} \rightarrow T^{2} X_{m}$ - линейное отображение из касательного пространства 1-го порлдка $T X_{m}$ в касательное пространство 2-го порядка $T^{2} X_{m}$. Причем для базисных векторов $\varepsilon_{i}: X_{m} \rightarrow T X_{m}$ линейное отображение $d \varepsilon_{i}: T X_{m} \rightarrow N_{1}^{2} \subset T^{2} X_{m}$ поднимает касательный вектор 1-го порядка в нормаль 2-го порядкa $N_{1}^{2}$.

Отображение $d \varepsilon_{i}$ всем касательным векторам ставит в соответствие векторы нормали 2-го порядка $N_{1}^{2}$. Такое расщепление соприкасающегося пространства $T^{2} X_{m}$ определяет простейшую (каноническую) аффинную связность $\stackrel{\circ}{\Gamma_{j k}^{i}}=-x_{j k}^{i}$ с формами $\stackrel{\circ}{\tilde{\omega}_{j}^{i}}=\left.\omega_{j}^{i}\right|_{\omega^{i}=0}$ (см. [10]). Эта связность является плоской и симметричной. Векторы $\tilde{\varepsilon}_{i j}=\dot{\varepsilon}_{i j}$ являются горизонтальными векторами для простейшей связности 1-го порядка (см. [10]), а нормаль $N_{1}^{2}$ - горизонтальным подпространством простейшей (канонической) связности, т.е. $H T^{2} X_{m}=N_{1}^{2}$ (см. $\left.[13,16]\right)$.

3. Нормали 3-го порядка. Обобщим равенство $\partial_{\varepsilon_{j}} \varepsilon_{i}=d \varepsilon_{i}\left(\varepsilon_{j}\right)$, справедливое для базисных касательных векторов 1-го порядка, на случай базисных касательных векторов 2-го порядка.

Теорема 2 (см. [12]). Для базисных касательных векторов 1-го и 2-го порядка $\varepsilon=\left\{\varepsilon_{i}\right\}$, $\varepsilon^{\prime}=\left\{\varepsilon_{i j}\right\}$ справедливы равенства

$$
\partial_{\varepsilon} \varepsilon^{\prime}=d \varepsilon^{\prime}(\varepsilon), \quad \partial_{\varepsilon^{\prime}} \varepsilon=d^{2} \varepsilon\left(\varepsilon^{\prime}\right), \quad \partial_{\varepsilon^{\prime}} \varepsilon^{\prime}=d^{2} \varepsilon^{\prime}\left(\varepsilon^{\prime}\right) .
$$

т.е. производные одних базисных векторов по направленияо данных базисных векторов 1-го (2-го) порлдка равны значениям дифберенииалов 1-го (2-го) порядка первых векторов на данных векmopax.

Вычисляя $\partial_{\varepsilon_{i}} \varepsilon_{p q}=\varepsilon_{i}\left(\varepsilon_{p q}\right)$ или действуя линейным отображением $d \varepsilon_{p q}$ на касательные векторы $\varepsilon_{i}$, получим следующие равные выражения:

$$
\begin{aligned}
\partial_{\varepsilon_{k}} \varepsilon_{i j} & =\stackrel{*}{x}_{i}^{s} \stackrel{*}{x}_{j}^{p} \stackrel{*}{*}_{k}^{q} \partial_{s p q}+x_{i j}^{s} \stackrel{*}{x}_{k}^{q} \stackrel{*}{x}_{s}^{p} \partial_{p q}, \\
d \varepsilon_{i j}\left(\varepsilon_{k}\right) & =\varepsilon_{i j k}-\varepsilon_{s} x_{i j k}^{s}-\dot{\varepsilon}_{s j} x_{i k}^{s}-\dot{\varepsilon}_{i s} x_{j k}^{s},
\end{aligned}
$$


которые обозначим $\partial_{\varepsilon_{k}} \varepsilon_{i j}=d \varepsilon_{i j}\left(\varepsilon_{k}\right)=\varepsilon_{i j, k}$.

Второй дифференциал касательных векторов $\varepsilon_{i}$, т.е. обычный дифференциал деривационной формулы (3) имеет вид (см. [11])

$$
d^{2} \varepsilon_{i}=\varepsilon_{i j k} \omega^{j} \omega^{k}+\varepsilon_{i j}\left(d \omega^{j}+\omega_{k}^{j} \omega^{k}\right)+2 \varepsilon_{j k} \omega^{k} \omega_{i}^{j}+\varepsilon_{j}\left(d \omega_{i}^{j}+\omega_{k}^{j} \omega_{i}^{k}+\omega^{k} \omega_{i k}^{j}\right) .
$$

Вычисляя $\partial_{\varepsilon_{p q}} \varepsilon_{i}=\varepsilon_{p q}\left(\varepsilon_{i}\right)$ или действуя линейным отображением $d^{2} \varepsilon_{i}$, определяемым по закону (4), на соприкасающиеся векторы $\varepsilon_{p q}$, получим следующие равные выражения:

$$
\begin{aligned}
\partial_{\varepsilon_{i j}} \varepsilon_{k} & =*_{i}^{s} \stackrel{*}{x}_{j}^{*} x_{k}^{*} \partial_{s p q}+x_{i j}^{s} \stackrel{*}{x}_{k}^{*} x_{s}^{*} \partial_{q p}, \\
d^{2} \varepsilon_{k}\left(\varepsilon_{i j}\right) & =\varepsilon_{k i j}-\varepsilon_{s} x_{k i j}^{s}-\dot{\varepsilon}_{s j} x_{k i}^{s}-\dot{\varepsilon}_{s i} x_{k j}^{s},
\end{aligned}
$$

которые обозначим $\partial_{\varepsilon_{i j}} \varepsilon_{k}=d^{2} \varepsilon_{k}\left(\varepsilon_{i j}\right)=\varepsilon_{k, i j}$.

Вычисляя $\partial_{\varepsilon_{k l}} \varepsilon_{i j}=\varepsilon_{k l}\left(\varepsilon_{i j}\right)$ или действуя линейным отображением $d \varepsilon_{i j}$ на соприкасающиеся векторы $\varepsilon_{k l}$, также получим равные выражения.

Поскольку слоевые координаты $x_{j k}^{i}, x_{j k l}^{i}$ симметричны по нижним индексам, то $\varepsilon_{i j, k}=\varepsilon_{k, i j}$, причем $\Delta \varepsilon_{i j, k}=\omega_{i j}^{l} \dot{\varepsilon}_{l k}$. Векторы $\dot{\varepsilon}_{i j}, \varepsilon_{k l, p}$ инвариантны в совокупности и определяют нормаль 3-го порядка $N_{1}^{3}=T^{3} X_{m} \backslash T X_{m}$ пространства $T X_{m}$, т.е. $N_{1}^{3}=\operatorname{span}\left(\dot{\varepsilon}_{i j}, \varepsilon_{k l, p}\right)$.

Векторы $\dot{\varepsilon}_{i j, k}=\varepsilon_{i j, k}-x_{i j}^{l} \dot{\varepsilon}_{k l}$ инвариантны сами по себе, причем для отображения, определяемого базисными векторами нормали 2-го порядка, имеем

$$
d \dot{\varepsilon}_{i j}: \varepsilon_{k} \in T X_{m} \rightarrow d \dot{\varepsilon}_{i j}\left(\varepsilon_{k}\right)=\dot{\varepsilon}_{i j, k} \in N_{2}^{3}=T^{3} X_{m} \backslash T^{2} X_{m} .
$$

Векторы $\dot{\varepsilon}_{i j}, \varepsilon_{i j, k}$ являются горизонтальными векторами для простейшей (канонической) связности 2-го порядка (см. $[10,11])$. Эта связность является плоской, а ее тензор кручения 2-го порядка равен нулю. Действительно, горизонтальные векторы 3 -го порядка

$$
\tilde{\varepsilon}_{i j k}=\varepsilon_{i j k}+\varepsilon_{l j} \Gamma_{i k}^{l}+\varepsilon_{i l} \Gamma_{j k}^{l}+\varepsilon_{l} \Gamma_{i j k}^{l}\left(\tilde{\varepsilon}_{i j k} \in T^{3} X_{m}\right)
$$

для простейшей аффинной связности 2 -го порядка $\stackrel{\circ}{\Gamma}{ }^{2}$ с компонентами

$$
\stackrel{\circ}{\Gamma}_{j k}^{i}=-x_{j k}^{i}, \quad \stackrel{\circ}{\Gamma}_{j k l}^{i}=-x_{j k l}^{i}+x_{s k}^{i} x_{j l}^{s}+x_{j s}^{i} x_{k l}^{s},
$$

имеют вид $\stackrel{\circ}{\tilde{\varepsilon}_{i j k}}=\varepsilon_{i j, k}$. Формы $\stackrel{\circ}{\tilde{\omega}}_{j k}^{i}=\left.\omega_{j k}^{i}\right|_{\omega^{i}=0}, \stackrel{\circ}{\tilde{\omega}}_{j}^{i}=\left.\omega_{j}^{i}\right|_{\omega^{i}=0}$ являются формами канонической связности 2-го порядка.

Для горизонтальных векторов простейшей связности 2-го порядка $\stackrel{\circ}{\Gamma}{ }^{2}$ справедливо разложение

$$
\stackrel{\circ}{\varepsilon}_{i j k}=x_{j k}^{l} \dot{\varepsilon}_{l i}+\dot{\varepsilon}_{j k, i}, \quad \stackrel{\circ}{\varepsilon}_{i j}=\dot{\varepsilon}_{i j}
$$

по базисным векторам $\dot{\varepsilon}_{i j} \in N_{1}^{2}$ и $\dot{\varepsilon}_{j k, i} \in N_{2}^{3}$.

Теорема 3. Пусть $U: X_{m} \rightarrow T^{2} X_{m}-$ векторное поле. Тогда $d U: T X_{m} \rightarrow T^{3} X_{m}-$ линейное отображсение из касательного пространства 1-го порядка $T X_{m}$ в касательное пространство 3го порядка $T^{3} X_{m}$. При этом для базисных векторов $\dot{\varepsilon}_{i j}: X_{m} \rightarrow N_{1}^{2} \subset T^{2} X_{m}$ нормали 2-го порлдка линейное отображение $d \dot{\varepsilon}_{i j}: T X_{m} \rightarrow N_{2}^{3} \subset T^{3} X_{m}$ поднимает касательный вектор 1-го порядка в нормаль 3-го порядка $N_{2}^{3}$ пространства $T^{2} X_{m}$.

Обобщая данный результат, можно утверждать, что отображения, задаваемые дифференциалами базисных векторов нормали $N_{r-1}^{r}$, переводят базисные касательные векторы 1-го порядка в базисные векторы нормали $N_{r}^{r+1}$.

\section{СПИСОК ЛИТЕРАТУРЫ}

1. Акивис M. А. Многомерная дифференциальная геометрия. - Калинин, 1977.

2. Бабурова О. В., Фролов Б. Н. Математические основы современной теории гравитации. - М.: Прометей, 2012.

3. Белова О. О. Грассманоподобное многообразие центрированных плоскостей// Мат. заметки. - 2018. -104 , № 6 . - С. $812-822$. 
4. Веблен О., Уайтхед Дж. Основания дифференциальной геометрии. - М.: ИЛ, 1949.

5. Восилюс P. В. Контравариантная теория дифференциального продолжения в модели пространства со связностью// Итоги науки и техн. Пробл. геом. - 1983. - 14. - С. 101-176.

6. Гурса Э. Курс математического анализа. - М.-Л.: ГИТТЛ, 1933.

7. Евтушик Л. Е., Лумисте Ю. Г., Остиану Н. М., Широков А. П. Дифференциально-геометрические структуры на многообразиях// Итоги науки и техн. Пробл. геом. - 1979. - 9. - С. 5-246.

8. Лаптев $Г$. $Ф$. Основные инфинитезимальные структуры высших порядков на гладком многообразии// Тр. геом. семин. ВИНИТИ. - 1966. - 1. - С. 139-189.

9. Полякова K. В. Двойственные методы исследования дифференциально-геометрических структур// Диффер. геом. многообр. фигур. - 2014. - 45. - С. 92-104.

10. Полякова К. В. Специальные аффинные связности 1-го и 2-го порядков// Диффер. геом. многообр. фигур. - 2015. - 46. - С. 114-128.

11. Полякова K. В. О задании аффинной связности 2-го порядка векторнозначными формами 1-го, 2-го и 3-го порядков// Диффер. геом. многообр. фигур. - 2016. - 47. - С. 108-125.

12. Полякова K. В. Тангенциальнозначные формы 2-го порядка// Мат. заметки. - 2019. - 105, № 1 . C. 84-94.

13. Ръбников А. К. Об аффинных связностях второго порядка// Мат. заметки. - 1981. - 29, № 2. C. $279-290$.

14. Ръбников A. К. Об обобщенных аффинных связностях второго порядка// Изв. вузов. Мат. - 1983. № 1. - C. $73-80$.

15. Сарданашвили Г. А. Геометрия и классические поля. Современные методы теории поля. Т. 1. - М.: YPCC, 1996.

16. Шевченко Ю. И. Оснащения голономных и неголономных гладких многообразий. - Калининград, 1998.

17. Catuogno P. A geometric Itô formula// Mat. Contemp. - 2005. - 33. - P. 85-99.

Полякова Катерина Валентиновна

Балтийский федеральный университет им. Иммануила Канта, Калининград

E-mail: KaPolyakova@kantiana.ru 